\title{
Management of acute COPD exacerbations in Australia: do we follow the guidelines?
}

\author{
Joyce L. Cousins (10) ${ }^{1,2,3}$, Richard Wood-Baker ${ }^{4}$, Peter A.B. Wark (10 ${ }^{3,5,6}$, \\ lan A. Yang ${ }^{7}$, Peter G. Gibson (103,5, Anastasia Hutchinson (10 ${ }^{8}$, Dimitar Sajkov? \\ Sarah A. Hiles ${ }^{1,3}$, Sameh Samuel $\mathbb{1}^{5,6}$ and Vanessa M. McDonald ${ }^{1,3,6}$
}

\begin{abstract}
Affiliations: ${ }^{1}$ School of Nursing and Midwifery, The University of Newcastle, Callaghan, Australia. ${ }^{2}$ Avondale University College, Faculty of Nursing, Wahroonga, Australia. ${ }^{3}$ Priority Research Centre for Healthy Lungs, Hunter Medical Research Institute, New Lambton Heights, Australia. ${ }^{4}$ School of Medicine, University of Tasmania, Hobart, Australia. ${ }^{5}$ School of Medicine and Public Health, The University of Newcastle, Callaghan, Australia. 'Dept of Respiratory and Sleep Medicine, Hunter Medical Research Institute, John Hunter Hospital, New Lambton Heights, Australia. ${ }^{7}$ The Prince Charles Hospital and University of Queensland, Chermside, Australia. ${ }^{8}$ School of Nursing and Midwifery, Deakin University, Geelong, Australia. ${ }^{9}$ Australian Respiratory and Sleep Medicine Institute, Flinders University and Flinders Medical Centre, Bedford Park, Australia.
\end{abstract}

Correspondence: Vanessa M. McDonald, Hunter Medical Research Institute, 1 Kookaburra Circuit, New Lambton Heights, 2305, Newcastle, Australia. E-mail: vanessa.mcdonaldanewcastle.edu.au

\section{ABSTRACT}

Objective: We aimed to assess adherence to the Australian national guideline (COPD-X) against audited practice, and to document the outcomes of patients hospitalised with an acute exacerbation of chronic obstructive pulmonary disease (COPD) at discharge and 28 days after.

Methods: A prospective clinical audit of COPD hospital admission from five tertiary care hospitals in five states of Australia was conducted. Post-discharge follow-up was conducted via telephone to assess for readmission and health status.

Results: There were 207 admissions for acute exacerbation (171 patients; mean 70.2 years old; 50.3\% males). Readmission rates at 28 days were $25.4 \%$, with one $(0.6 \%)$ death during admission and eight (6.1\%) post-discharge within 28 days. Concordance to the COPD-X guidance was variable; $22.7 \%$ performed spirometry, $81.1 \%$ had blood gases collected when forced expiratory volume in $1 \mathrm{~s}$ was $<1 \mathrm{~L}$, 99.5\% had chest radiography performed, $95.1 \%$ were prescribed systemic corticosteroids and $95 \%$ were prescribed antibiotic therapy. There were $89.1 \%$ given oxygen therapy and $92.6 \%$ when arterial oxygen tension was < $80 \mathrm{mmHg} ; 65.6 \%$ were given ventilatory assistance when $\mathrm{pH}$ was $<7.35$. Only $32.4 \%$ were referred to pulmonary rehabilitation but $76.8 \%$ had general practitioner follow-up arranged.

Conclusion: When compared against clinical practice guidelines, we found important gaps in management of patients admitted with COPD throughout tertiary care centres in Australia. Strategies to improve guideline uptake are needed to optimise care.

$@$ ERSpublications

A multisite audit of hospital admissions for patients experiencing \#AECOPD in Australia highlighted important gaps in management when compared against national guidelines. Further focus on how we can improve guideline uptake by clinicians is needed. http://bit.ly/2 VjGnHw

Cite this article as: Cousins JL, Wood-Baker R, Wark PAB, et al. Management of acute COPD exacerbations in Australia: do we follow the guidelines? ERJ Open Res 2020; 6: 00270-2019 [https:// doi.org/10.1183/23120541.00270-2019]. 


\section{Introduction}

Chronic obstructive pulmonary disease (COPD) is a complex and heterogeneous disease that is responsible for a major burden of illness. In Australia, $7.5 \%$ of people $\geqslant 40$ years old have COPD of Global Initiative for Obstructive Lung Disease (GOLD) stage II or above, increasing to nearly $30 \%$ in the $>75$-years age group [1]. COPD is the fifth leading cause of death in Australia [2] and World Health Organisation estimates predict that COPD will become the third leading cause of death worldwide by 2030 [3].

Acute exacerbations of COPD (AECOPD) are common occurrences, characterised by a deterioration of respiratory symptoms that necessitates additional therapy [4]. This deterioration affects patients' health status, and contributes to disease progression, increased rates of hospitalisation and death [4]. AECOPD make up the highest share of the total COPD economic burden [4], expending significant healthcare resources [5]. COPD is the leading cause of avoidable hospital admissions amongst chronic disease in Australia, with $>78000$ potentially preventable admissions in 2016-2017 [6]. Additionally, Australia has one of the highest rates of hospital admissions for COPD within the Organisation for Economic Co-operation and Development [7]. These data highlight the importance of using the latest evidence to minimise AECOPD, reduce future risks and prevent hospitalisation.

Guidelines facilitate the incorporation of research evidence into clinical practice and support clinicians in their patient care decisions [8]. The Australian and New Zealand COPD-X guidelines seek to facilitate changes in clinical practice based on sound evidence [9]. However, guideline utilisation in clinical practice can be challenging and uptake remains poor [8], with previous national audits of practice $[10,11]$ and international audits [12-14] demonstrating variable adherence to evidence-based guidelines in the management of AECOPD.

The aims of this study were to collect prospective data on the management of hospital admissions for AECOPD across multiple sites in Australia and to compare contemporary management against a best practice guideline (COPD-X) [9]. We hypothesised that there would be discordance in the adherence to these guidelines and that the level of adherence would be similar across tertiary care sites.

\section{Methods}

A prospective clinical audit of AECOPD hospital admissions was conducted in tertiary care hospitals in five of the eight states/territories of Australia: Flinders Medical Centre (South Australia), John Hunter Hospital (New South Wales), Northern Health (Victoria), Royal Hobart Hospital (Tasmania) and The Prince Charles Hospital (Queensland). At 28 days post-discharge, participants were followed-up by telephone to determine readmission events and health status.

Institutional ethics approval was gained for all sites participating in the study (lead site Hunter New England Human Research Ethics Committee reference number 12/10/17/5.05) and all participants provided written informed consent.

\section{Audit tool}

To reflect the Australian context, a previously validated clinical audit tool was modified from the UK COPD tool $[10,15]$. Core data collected included patient characteristics, comorbidities, patient accommodation, vital signs, chest radiographic appearance, pharmacological interventions, respiratory status or support required, and spirometry results. Health status was assessed using the COPD Assessment Test (CAT) [16], measured during admission and at 28 days post-discharge by telephone follow-up. Data were collected between October 2012 and April 2013, and were entered into a purpose-built, web-based database. Data were assessed against the COPD-X guidelines to determine concordance. These guidelines have been available to clinicians for use since 2003 [17] and are updated quarterly; variables that we measured concordance against had not undergone any recommendation changes in the preceding 12 months. For the purpose of this audit, version 2.34 (November 2012) was utilised [9].

\section{Participants}

Each centre prospectively identified admissions with AECOPD to their healthcare facility, using methods most appropriate to the individual site. Patients admitted with an AECOPD were approached and invited to participate. Inclusion and exclusion criteria are presented in table 1.

\section{Data analysis}

Analyses were performed using Stata 14 (Stata Corporation, College Station, TX, USA). Categorical data were described using frequencies and percentages. Continuous data were described using means and standard deviations for normally distributed data, and medians and interquartile ranges (IQRs) for non-normally distributed data. Associations between categorical variables were investigated using Chi-squared and Fisher's exact tests, with continuous data compared using Student's t-test or Wilcoxon 


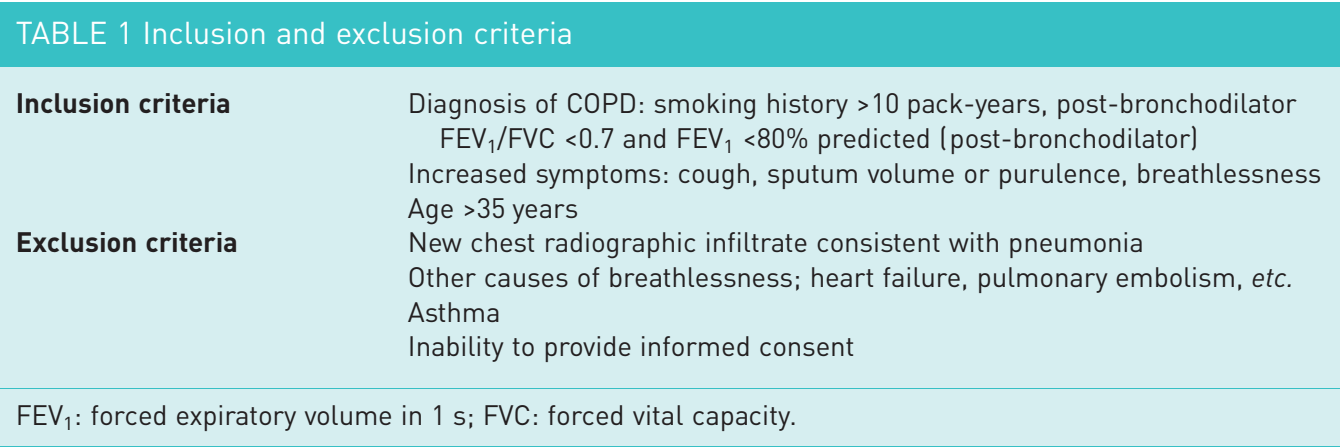

signed rank. Comparison between the treatment sites were investigated with ANOVA for normally distributed data, with Bonferroni-corrected post hoc comparisons conducted when required. Kruskal-Wallis was used for non-normally distributed data. The relationship between selected variables and readmission were investigated using logistic regression, and length of stay via negative binomal regression and linear regression as appropriate. p-values of $<0.05$ were considered significant.

Venous blood gas results were transformed by adding 0.03 to the venous $\mathrm{pH}$ result as summarised in the review by BYRne et al. [18].

\section{Results}

\section{Demographic characteristics of patient population}

Data were collected for 207 admissions in 171 patients with AECOPD. Table 2 presents demographic data, select clinical parameters and admission outcomes for each of the study sites. Most patients had a diagnosis of COPD prior to their admission. The mean \pm SD age of patients was $70.2 \pm 9.9$ years and $50.3 \%$ were male. Most were former smokers, with current smokers representing approximately one third. Over half lived with another person. Patients had a mean \pm SD of $3.7 \pm 2.0$ comorbidities. Patient characteristics were generally similar across sites.

Almost all patients reported an increased level of breathlessness and around half reported a change in sputum colour prior to admission. Nearly three quarters had been hospitalised for COPD within the previous 24 months. There was one death during hospitalisation and eight (6.1\%) within 28 days following discharge. The median length of stay (LOS) was 6 days (IQR 3-9 days), with significant differences seen in the LOS between sites 1 and $2(\mathrm{p}<0.001)$ and sites 1 and $4(\mathrm{p}<0.001)$.

Readmissions within 28 days occurred in 36 patients (25.4\%) and these patients had more comorbidities (4.6 \pm 2.2 versus $3.5 \pm 1.8, \mathrm{p}=0.002$ ). CAT score was not found to predict death or readmission. In this cohort, it was associated with LOS. For each unit increase in CAT score at discharge, there was an increase of 0.17 days in LOS ( $\mathrm{p}=0.044 ; 95 \%$ CI $0.004-0.343$ ). At 28 days, the CAT score was significantly better than at discharge $(21.6 \pm 7.9$ versus $24.5 \pm 6.9, \mathrm{p}<0.001)$; however, the discharge score did not differ significantly between those who were and were not readmitted within 28 days (mean $26.4 \pm 7.0$ versus $24.0 \pm 6.3, \mathrm{p}=0.79)$.

A quarter of patients were discharged home on oxygen, with significant differences observed between sites $(\mathrm{p}=0.016)$. Of these, seven $(19.4 \%)$ out of 36 patients had been prescribed domiciliary oxygen for the first time during the audited admission, with differences seen between the sites $(\mathrm{p}=0.037)$; most ( 28 out of $35,80 \%)$ were prescribed oxygen via a concentrator $(\mathrm{p}=0.129)$ and over a quarter of patients discharged on oxygen (nine out of 34, 26.5\%; $\mathrm{p}=0.018$ ) received follow up in an oxygen clinic. However, of those who did not have follow up in an oxygen clinic, $83.3 \%$ ( 25 out of $30, \mathrm{p}=0.033$ ) had follow up with a respiratory physician. In many cases, if not all, this review would have included an assessment of long-term oxygen therapy requirements.

\section{Concordance to COPD-X guidelines}

Table 3 outlines the COPD-X management recommendations, and summarises the overall and site-specific concordance against a selection of these recommendations.

Spirometry was performed in only $22.7 \%$ of admissions. There were significant differences $(\mathrm{p}<0.001)$ and wide variation between sites (range 1.5\%-53.2\%). Of the patients who had forced expiratory volume in $1 \mathrm{~s}$ $\left(\mathrm{FEV}_{1}\right)$ documented, 106 had an $\mathrm{FEV}_{1}<1.0 \mathrm{~L}$, with $81.1 \%$ of these having an arterial blood gas (ABG) or venous blood gas $(\mathrm{VBG})$ collected $(\mathrm{p}=0.017)$. Overall, 156 patients $(75.4 \%)$ had either an ABG or a VBG taken at some point during their admission. There was a high level of concordance with chest radiography, performed in $99.5 \%$ within the first $24 \mathrm{~h}(\mathrm{p}=0.039)$. 
TABLE 2 Patient characteristics and key clinical indicators

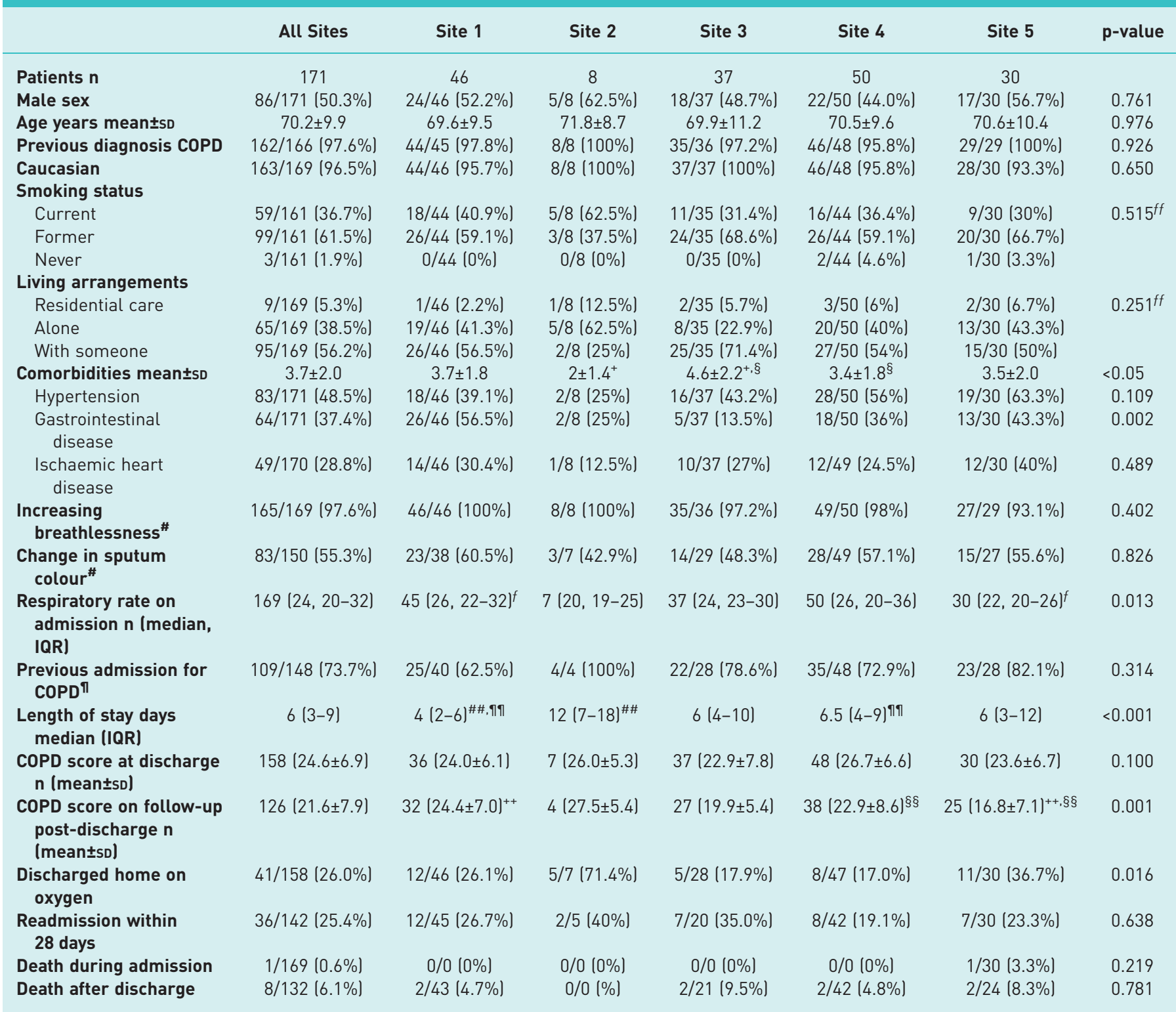

Data are presented as $\mathrm{n} / \mathrm{N}(\%)$, where $\mathrm{N}$ is the number of data available for analysis and $\%$ are is documented values lunrecorded, unknown and undocumented values have been treated as missing values), unless otherwise stated. Data are calculated on 171 patients. IQR: interquartile range. ${ }^{\#}$ : prior to admission; ${ }^{\text {I }}$ : in previous 24 months. ${ }^{+}$: significant difference between sites 2 and 3 ( $p=0.006$ ); ${ }^{\S}$ : significant difference between sites 3 and $4(p=0.043)_{;}{ }^{f}$ : significant difference between sites 1 and $5(p=0.013) ;{ }^{\# \#}$ : significant difference between sites 1 and 2 ( $p \leqslant 0.001) ; ~$ ๆा: significant difference between sites 1 and $4(p \leqslant 0.001) ;{ }^{++}$: significant difference between sites 1 and 5 ( $\left.p=0.002\right)$; $\S \S$ : significant difference between sites 4 and $5(p=0.020) ;{ }^{f f}$ : overall $p$-value.

The prescription of systemic corticosteroids was high (range 90-100\%, $\mathrm{p}=0.085$ ). Antibiotic prescription for those with a documented change in sputum colour was also high overall (95.0\%), although lower at site 2 compared to other sites $(66.7 \%, \mathrm{p}=0.085)$. However, most patients with no change in the colour $(85.7 \%)$ or volume $(84.9 \%)$ of their sputum were also prescribed antibiotics.

Pulse oximetry data were not recorded during this audit, so appropriate use of oxygen could not be determined. However, oxygen was administered to $89.1 \%$ of patients, with only $27 \%$ of these having a written oxygen prescription (range 3.2-76.9\%, $\mathrm{p}<0.001$ ). Overall, 32 patients had a $\mathrm{pH}<7.35$, with ventilatory assistance used in 21 (65.6\%). Of the 18 patients who had the type of ventilatory assistance documented, it is clear that three received invasive ventilation. Reasons for not using noninvasive ventilation (NIV) or invasive ventilation included a documented decision not to escalate to NIV or invasive ventilation (18.2\%), "patient responded to medical therapy" (45.6\%) and in 36.4\%, no reason was listed. 
TABLE 3 Observed frequencies for each admission for management of acute exacerbation of COPD according to COPD-X guideline recommendations

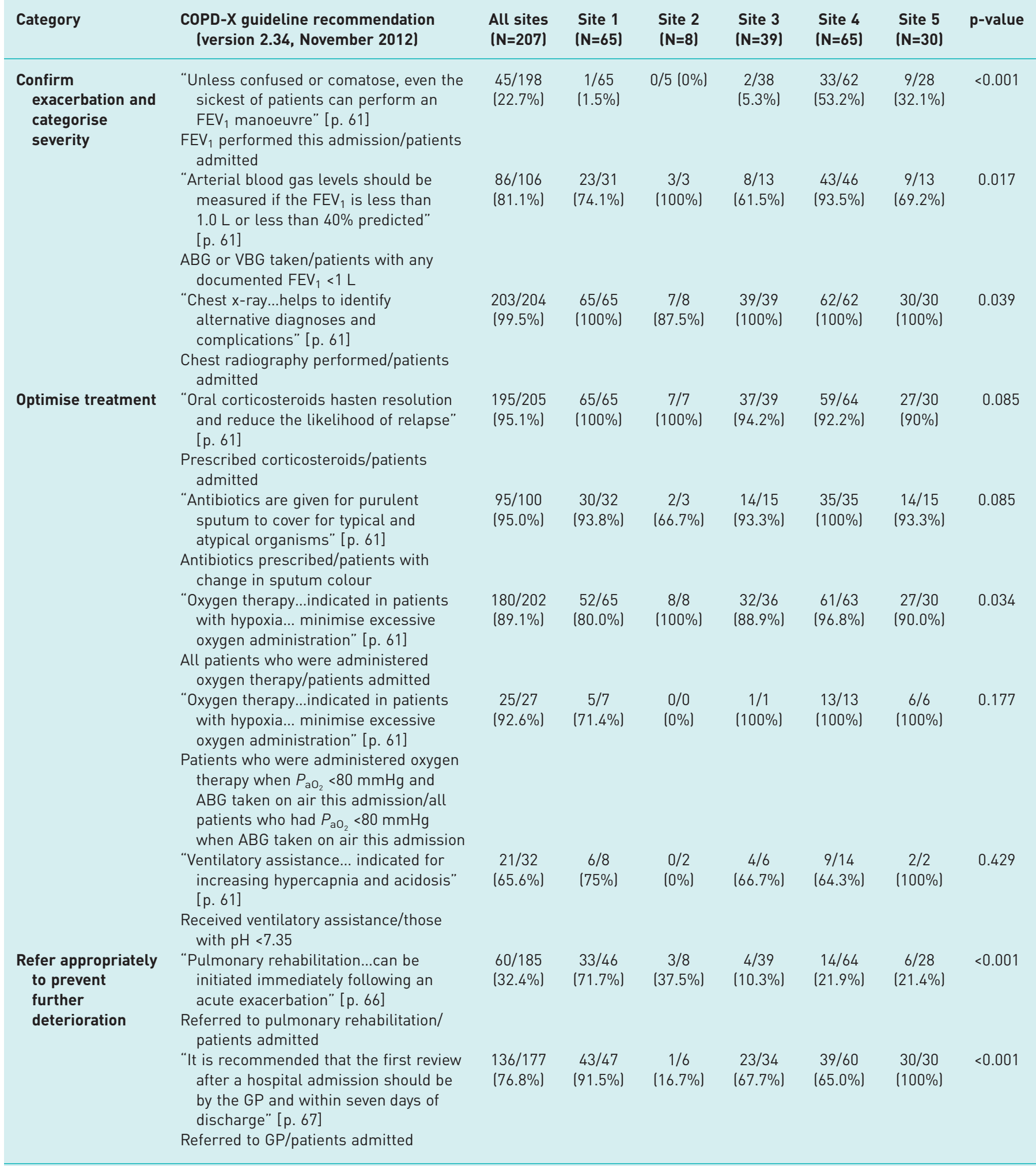

Data calculated on individual admissions ( $\mathrm{n}=207$ ); \% are for documented values (unrecorded, unknown and undocumented values have been treated as missing values). $\mathrm{FEV}_{1}$ : forced expiratory volume in $1 \mathrm{~s} ; \mathrm{ABG}$ : arterial blood gas; VBG: venous blood gas; $P_{\mathrm{aO}_{2}}$ : arterial oxygen tension; GP: general practitioner. 
Overall, $76.8 \%$ of patients had general practitioner follow-up arranged prior to discharge; however, there was wide variation between sites (range $16.7-100 \%, \mathrm{p}<0.001$ ). Referral to pulmonary rehabilitation was low in most centres (mean 32.4\%, range 10.3-71.7\%; $\mathrm{p}<0.001$ ).

We examined the impact of concordance on LOS and readmission for recommendations where there was $<75 \%$ concordance in the COPD-X categories "confirming exacerbation/categorising severity" (performance of $\mathrm{FEV}_{1}$ ) and "optimising treatment" (receiving ventilatory assistance). Neither performance of $\mathrm{FEV}_{1}$ (incident rate ratio (IRR) $1.36, \mathrm{p}=0.185$; 95\% CI $0.862-2.156$ ) or receiving ventilatory assistance (IRR 1.196, $\mathrm{p}=0.217 ; 95 \%$ CI $0.900-1.589$ ) were significantly associated with LOS. In addition, there was no statistically significant difference $(\mathrm{p}=0.090)$ between patients who did or did not receive ventilatory support on readmission to hospital. However, patients who did not perform spirometry as part of the audited admission were 3.6 times more likely to be readmitted ( $p=0.007,95 \%$ CI $1.413-9.173$ ); $14.3 \%$ of those who performed a spirometry were readmitted, compared to $37 \%$ of those who did not perform a spirometry during their admission.

\section{Discussion}

This prospective study of five tertiary care facilities across Australia demonstrated that there are variations in concordance to the COPD-X guideline for inpatient management of patients with AECOPD. Interventions with high concordance included performance of chest radiography, provision of treatment with oral corticosteroids and antibiotics, and administration of oxygen therapy. Discordance was seen to the recommendations of performing spirometry and referral to pulmonary rehabilitation, with rates varying substantially across sites. This study reflects actual clinical practice and has provided insight into the gaps in AECOPD management when compared to evidenced-based guidelines.

Patient characteristics were similar to those of other audits $[10,11,19]$. The median LOS in this group was 6 days. Our data are similar to previous Australian studies $[10,11]$ that reported a median LOS of 5 days, but shorter than international data where median LOS has been reported between 7 and 8 days [20-22]. The reason for the significant difference seen in LOS between Australian sites is not clear but may be related to variations in management or local protocols. CAT score was shown to impact LOS, demonstrating its value as a reportable patient outcome for use during hospital admission.

Smoking rates, while similar to other Australian data $[10,11]$, were higher than those reported by European studies [21, 22] ( $9 \%$ and 30\%, respectively). Smoking rates in the Australian cohort are high when compared to the national average $(9.3 \%)$ in the 65-75-year age group [23], highlighting the need for effective smoking cessation strategies for patients with COPD.

Our data support previous national [10,19] and international [24] findings demonstrating underutilisation of spirometry. These results compare to previous national audits. A study by KHIALANI et al. [19] reported a measured $\mathrm{FEV}_{1}$ in $17.2 \%$ of patients, while PRETTO et al. [10] reported spirometry being recorded in $29 \%$. COPD-X suggest that spirometry during admission is of value in assessing severity of an AECOPD [9], and may be useful in confirming diagnosis and assessing severity and clinically useful for ongoing management [25] In our audit, not having spirometry performed during the admission increased the odds of 28-day readmission.

Several studies have demonstrated that valid and reproducible spirometry traces can be obtained in patients admitted with AECOPD, and is important in confirming the presence of airflow limitation where diagnosis of COPD is not confirmed [26, 27]. Poor spirometry uptake may be due to differences in recommendations by key guidelines on the need for spirometry testing during admission $[4,28]$. While COPD-X argues that "even the sickest of patients can perform an $\mathrm{FEV}_{1}$ manoeuvre" [28], the GOLD guidelines [4] do not routinely recommend that sick patients perform spirometry. It is also important to consider the practicality or capacity of emergency department staff to obtain acceptable spirometry traces. Patient admissions are not restricted to "office hours" when lung function laboratories are open. In addition, appropriate equipment and staff who are skilled in obtaining accurate measurements may not always be available at the times when patients present to the emergency department. However, acute care services need to be improving diagnostic accuracy in the assessment of COPD severity and demonstrating the importance of this diagnostic tool to primary care practitioners. Any admission to acute care services can present an opportunity for reassessment and classification of the severity of airflow limitation to guide decisions on appropriate treatment, particularly as poor use of spirometry in primary care has been shown to be associated with under and overdiagnosis of COPD patients [29].

COPD-X [9] recommend measurement of ABGs if the $\mathrm{FEV}_{1}$ is $<1.0 \mathrm{~L}$ or $40 \%$ predicted, or when oxygen saturations are $<90 \%$. Our study did not record peripheral oxygen saturation data and rates of spirometry on admission were low; however, while three quarters of all patients had either an ABG or VBG collected (156 patients, $75.3 \%$ ), $18.9 \%$ of admissions who had a $\mathrm{FEV}_{1}<1.0 \mathrm{~L}$ did not have blood gases taken. The reason(s) not to perform these measurements are unknown and our study was not designed to identify 
these, but these data highlights gaps in management. Early identification of hypercapnic respiratory failure is important, to identify both those at highest risk of death during admission and those who will benefit from treatment with NIV [5]. Failure to obtain blood gas measurements may lead to missed opportunities to provide NIV to those in acute respiratory failure. Blood gas collection occurred in $81.1 \%$ for those with a $\mathrm{FEV}_{1}<1.0 \mathrm{~L}$ and in $75.3 \%$ of patients overall. Results compared to other Australian studies where collection rates (ABG or VBG) ranged between $62 \%$ and $77 \%[10,19,30,31]$. They also compare favourably to international literature from South East Asia, where rates of blood gas collection occurred in less than a quarter of patients admitted to the emergency department [31]. However, blood gas collection is consistently higher in Europe, with documented rates between $80 \%$ and $90 \%[13,21,22]$.

NIV was indicated in 32 patients, with 21 receiving ventilatory assistance. National $[10,19,31]$ and international $[13,31]$ data highlight missed opportunities for the use of NIV in patients with acidosis. This equates to $11(34 \%)$ patients who could have potentially benefited from this therapy. High-quality evidence now exists to support the use of NIV in addition to usual care for patients with acute hypercapnic respiratory failure; interestingly, the number needed to avoid one death is 12 , very close to the number in our audit missing out on treatment [32]. NIV has been shown to reduce mortality by up to $46 \%$, reduce the need for intubation by $65 \%$ and, on average, reduces the LOS for these patients by 3.5 days [32]. Our results confirm that NIV is underutilised.

Excellent concordance was seen for the recommendation to perform a chest radiograph, with almost $100 \%$ adherence seen in our study. Both national $[10,19,30,31]$ and international $[13,21,31]$ data consistently demonstrate high concordance ( $>90 \%$ across studies) with this recommendation. Our study also demonstrates high concordance across all sites (range 90-100\%) for corticosteroid prescription. These results are higher than those reported by РRETTO et al. [10]. It also compares favourably to international data that demonstrate concordance rates between $82.3 \%$ [13] and 92\% [21].

In most admissions (95.0\%) audited, patients were prescribed antibiotics when there was evidence of change in sputum colour. These compare favourably to the study by PRETTO et al. [10] but lower prescription rates were noted in studies that have examined Australian emergency department management of patients with AECOPD, where rates ranged between 58\% and 75\% [19, 30, 31]. Guidelines [9] recommend antibiotic cover for patients with purulent sputum. This audit did not specifically investigate presence of purulent sputum; however, it did ask if a change in sputum colour was noted. Our study also demonstrated high rates of antibiotic prescription in patients who had no documented change in sputum colour $(85.7 \%)$. This indicates a potential overprescription of antibiotics in this group. TANG et al. [11] identified that $45 \%$ of patients were overprescribed antibiotic therapy. A number of studies have identified inappropriate prescription of antibiotic therapy in the AECOPD patient cohort [12, 33, 34]. Furthermore, a 2018 Cochrane review concluded that aside from AECOPD patients who are treated in an intensive care unit, the effects of antibiotic therapy use in this patient group is small [35]. Antibiotic resistance is a major public health problem [36], one contributing factor associated with the emergence of multidrug-resistant bacteria is inappropriate prescription of antibiotics [37]. Our data demonstrates that improvement is needed to ensure antibiotics are prescribed in those who would most benefit.

Rates of oxygen use during an acute exacerbation are high in both national $[10,11]$ and international $[13$, 21] literature within the COPD cohort, with its use recommended for those who are hypoxic. Similarly, oxygen use across all sites was high in our audit. It was difficult to assess the appropriateness of delivery in the absence of more specific clinical data. The written prescription of oxygen differed significantly between sites, with rates of prescription poor (3.2\%) at two sites while significantly better rates observed at another (76.9\%). Reasons for these differences in prescription rates are not clear from the data collected. The practice of prescribing oxygen therapy is poor internationally, with many reasons offered in the literature and multiple interventions trialled in efforts to improve this practice [38]. However, quality improvement projects in the UK have demonstrated improvements in the practice of oxygen prescription, the 2017-2018 audit demonstrating that $72 \%$ of admissions requiring oxygen having a prescription [39]. Notably, the Thoracic Society of Australia and New Zealand (TSANZ) did not release their clinical practice guidelines on oxygen use in adults until 2015, which was after the current audit was undertaken (2012-2013). However, the British Thoracic Society guidance [40] advocating for oxygen prescription has been published since 2008 .

Less than a third of patients were referred to pulmonary rehabilitation. While this result is not unexpected or particularly poor, when comparing this to current guidance that asks clinicians to "consider pulmonary rehabilitation at any time following an exacerbation" [41], a rate of $32 \%$ certainly demonstrates room for improvement given the evidence that supports the efficacy of this intervention $[42,43]$. Considering the metropolitan location of all study sites, one could reasonably expect to find the largest number of and easiest access to pulmonary rehabilitation programmes. The low referral rates demonstrate that there is 
scope to improve referral to pulmonary rehabilitation. Both national $[10,11]$ and international [24] data have consistently demonstrated low rates of referral to pulmonary rehabilitation programmes despite high-quality evidence demonstrating it improves quality of life, exercise capacity and reduces healthcare utilisation [42]. The TSANZ pulmonary rehabilitation guideline recommends provision of pulmonary rehabilitation within 2 weeks of discharge and efforts to improve referral are needed [43]. General practitioner follow-up was high overall $(76.8 \%)$; however, significant differences were seen in referral rates between sites (range 16.7\%-100\%).

This study presented several strengths and limitations. To our knowledge, this is the first national audit conducted in multiple sites across five Australian states. This provides a representative sample from an Australia tertiary care perspective. The eligibility criteria were broad and inclusive, in order to reflect clinical practice. The prospective study design avoided the inclusion of patients whose diagnostic codes were incorrect, as only patients who had been assessed by a clinician as having an AECOPD were included. This design, however, also presents some limitations. While patients were identified prospectively, data were recorded from medical records, which led to some missing and/or inconsistently coded variables. Poor documentation of patient data in the patient notes may have led to perceived poor concordance even where none may have existed in practice. In addition, this was a real-world clinical audit conducted as part of usual clinical care. The real-world nature of this study meant that there were differences in the levels of data acquisition between sites.

Overall, important gaps in management were seen nationally when compared against the COPD-X guideline. There is scope for improvements in performance of $\mathrm{FEV}_{1}$ and referral to pulmonary rehabilitation programmes. In addition, increased utilisation of NIV may reduce mortality and endotracheal intubation. Strategies that have demonstrated improvements in the quality of care for AECOPD have been highlighted in the literature and deserve serious consideration for inclusion within the Australian context. In the UK, continuous clinical audit was commenced in 2017 for COPD care. This strategy has seen real improvements in the quality of care received by patients admitted with AECOPD [39]. In addition, strategies such as the use of care bundles have been shown to decrease readmission rates in the AECOPD patient group [44]. Further exploration around the feasibility of such strategies is needed in Australia to improve best evidence uptake, clinical practice and patient outcomes.

\section{Conclusion}

This study found important gaps in the management of patients admitted with COPD throughout tertiary care centres in Australia and variation in concordance with clinical practice guidelines between centres. Further focus on how we can improve the uptake of guidelines by clinicians is needed.

Conflict of interest: J.L. Cousins has nothing to disclose. R. Wood-Baker has nothing to disclose. P.A.B. Wark has nothing to disclose. I.A. Yang has nothing to disclose. P.G. Gibson reports a Practitioner Fellowship from the NHMRC participation in educational symposia and in studies funded by AstraZeneca and by GlaxoSmithKline; and participation in educational symposia funded by Boehringer Ingelheim and by Novartis, all outside the submitted work. A. Hutchinson has nothing to disclose. D. Sajkov has nothing to disclose. S.A. Hiles reports salary from grants paid to her institution (University of Newcastle) by GlaxoSmithKline and AstraZeneca outside the submitted work. S. Samuel has nothing to disclose. V.M. Mcdonald reports grants and personal fees from AstraZeneca and GSK, and personal fees from Menarini, outside the submitted work

\section{References}

1 Toelle BG, Xuan W, Bird TE, et al. Respiratory symptoms and illness in older Australians: the Burden of Obstructive Lung Disease (BOLD) study. Med J Aust 2013; 198: 144-148.

2 Australian Institute of Health and Welfare. Australia's health 2018. Australia's health series no. 16 Cat. no. 221. Canberra, AIHW, 2018.

3 World Health Organisation (WHO). Chronic respiratory diseases. Burden of COPD. 2019. www.who.int/ respiratory/copd/burden/en/. Date last accessed: July 19, 2019.

4 GOLD. Global Strategy for the Diagnosis, Prevention, and Management of Chronic Obstructive Pulmonary Disease. (2019 Report). https://goldcopd.org/wp-content/uploads/2018/11/GOLD-2019-v1.7-FINAL-14Nov2018WMS.pdf.

5 Ko FW, Chan KP, Hui DS, et al. Acute exacerbation of COPD. Respirology 2016; 21: 1152-1165.

6 Australian Institute of Health and Welfare. Admitted patient care 2016-17: Australian hospital statistics. Canberra, AIHW, 2018.

7 OECD. Health at a Glance 2017: OECD Indicators. Paris, OECD Publishing, 2017. https://doi.org/10.1787/health glance-2017-en.

8 Gagliardi AR, Brouwers MC, Palda VA, et al. How can we improve guideline use? A conceptual framework of implementability. Implement Sci 2011; 6: 26.

9 Abramson M, Crockett AJ, Dabscheck E, et al. The COPDX Plan: Australian and New Zealand Guidelines for the management of Chronic Obstructive Pulmonary Disease. Version 2.34 ed. Brisbane, Lung Foundation Australia, 2012.

10 Pretto JJ, McDonald VM, Wark PA, et al. Multicentre audit of inpatient management of acute exacerbations of chronic obstructive pulmonary disease: comparison with clinical guidelines. Intern Med J 2011; 42: 380-387. 
11 Tang CY, Taylor NF, McDonald CF, et al. Level of adherence to the GOLD strategy document for management of patients admitted to hospital with an acute exacerbation of COPD. Respirology 2014; 19: 1191-1197.

12 Lopez-Campos JL, Hartl S, Pozo-Rodriguez F, et al. Antibiotic prescription for COPD exacerbations admitted to hospital: European COPD Audit. PLoS One 2015; 10: e0124374.

13 Roberts CM, Lopez-Campos JL, Pozo-Rodriguez F, et al. European hospital adherence to GOLD recommendations for chronic obstructive pulmonary disease (COPD) exacerbation admissions. Thorax 2013; 68: 1169-1171.

14 Stone RA, Holzhauer-Barrie J, Lowe D, et al. COPD: Who cares matters. National Chronic Obstructive Pulmonary Disease (COPD) Audit Programme: Clinical audit of COPD exacerbations admitted to acute units in England and Wales 2014. National clinical audit report.. London, Royal College of Physicians, 2015.

15 Roberts CM, Ryland I, Lowe D, et al. Audit of acute admissions of COPD: standards of care and management in the hospital setting. Eur Respir J 2001; 17: 343-349.

16 Jones PW, Harding G, Berry P, et al. Development and first validation of the COPD Assessment Test. Eur Respir J 2009; 34: 648-654

17 McKenzie DK, Frith PA. The COPDX Plan: Australian and New Zealand Guidelines for the management of Chronic Obstructive Pulmonary Disease 2003. Med J Aust 2003; 178: S1-S39.

18 Byrne AL, Bennett M, Chatterji R, et al. Peripheral venous and arterial blood gas analysis in adults: are they comparable? A systematic review and meta-analysis. Respirology 2014; 19: 168-175.

19 Khialani B, Sivakumaran P, Keijzers G, et al. Emergency department management of acute exacerbations of chronic obstructive pulmonary disease and factors associated with hospitalization. J Res Med Sci 2014; 19: 297-303.

20 European Respiratory Society. An international comparison of COPD care in Europe: Results of the first European COPD audit. Sheffield, European Respiratory Society, 2012.

21 Pozo-Rodriguez F, Lopez-Campos JL, Alvarez-Martinez CJ, et al. Clinical audit of COPD patients requiring hospital admissions in Spain: AUDIPOC study. PLoS One 2012; 7: e42156.

22 Escarrabill J, Torrente E, Esquinas $\mathrm{C}$, et al. Clinical audit of patients hospitalized due to COPD exacerbation. MAG-1 study. Arch Bronconeumol 2015; 51: 483-489.

23 ABS. Australian Health Survey: Updated Results, 2011-12. Canberra, Australian Bureau of Statistics, 2013.

24 Lodewijckx C, Sermeus W, Vanhaecht $\mathrm{K}$, et al. Inhospital management of COPD exacerbations: a systematic review of the literature with regard to adherence to international guidelines. J Eval Clin Pract 2009; 15: 1101-1110.

25 Rea H, Kenealy T, Adair J, et al. Spirometry for patients in hospital and one month after admission with an acute exacerbation of COPD. Int J Chron Obstruct Pulmon Dis 2011; 6: 527-532.

26 Prieto Centurion V, Huang F, Naureckas ET, et al. Confirmatory spirometry for adults hospitalized with a diagnosis of asthma or chronic obstructive pulmonary disease exacerbation. BMC Pulm Med 2012; 12: 73.

27 Fernández-Villar A, Represas-Represas C, Mouronte-Roibás C, et al. Reliability and usefulness of spirometry performed during admission for COPD exacerbation. PLoS One 2018; 13: e0194983.

28 Yang IA, Brown JL, George J, et al. The COPD-X Plan: Australian and New Zealand Guidelines for the management of Chronic Obstructive Pulmonary Disease 2018. Version 2.55, August 2018. Brisbane, Lung Foundation Australia, 2018.

29 Heffler E, Crimi C, Mancuso S, et al. Misdiagnosis of asthma and COPD and underuse of spirometry in primary care unselected patients. Respir Med 2018; 142: 48-52.

30 Gerber A, Moynihan C, Klim S, et al. Compliance with a COPD bundle of care in an Australian emergency department: a cohort study. Clin Respir J 2018; 12: 706-711.

31 Kelly AM, Holdgate A, Keijzers G, et al. Epidemiology, treatment, disposition and outcome of patients with acute exacerbation of COPD presenting to emergency departments in Australia and South East Asia: an AANZDEM study. Respirology 2018; 23: 681-686.

32 Osadnik CR, Tee VS, Carson-Chahhoud KV, et al. Non-invasive ventilation for the management of acute hypercapnic respiratory failure due to exacerbation of chronic obstructive pulmonary disease. Cochrane Database Syst Rev 2017; 7: CD004104.

33 Australian Commission on Safety and Quality in Health Care. Antimicrobial prescribing practice in Australian hospitals: results of the 2014 National Antimicrobial Prescribing Survey. Sydney, ACSQHC, 2015.

34 McGoldrick C, Ulahannan T, Krebs KL. Review of antibiotic use in respiratory disorders at a regional hospital in Queensland. Collegian 2016; 23: 391-395.

35 Vollenweider DJ, Frei A, Steurer-Stey CA, et al. Antibiotics for exacerbations of chronic obstructive pulmonary disease. Cochrane Database Syst Rev 2018; 10: CD010257.

36 Davey P, Marwick CA, Scott CL, et al. Interventions to improve antibiotic prescribing practices for hospital inpatients. Cochrane Database Syst Rev 2017; 2: CD003543.

37 Ventola CL. The antibiotic resistance crisis: part 1: causes and threats. PT 2015; 40: 277-283.

38 Cousins JL, Wark PAB, McDonald VM. Acute oxygen therapy: a review of prescribing and delivery practices. Int J Chron Obstruct Pulmon Dis 2016; 11: 1067-1075.

39 Hurst J, McMillan V, Mortier K, et al. National Asthma and Chronic Obstructive Pulmonary Disease Audit Programme (NACAP). COPD clinical audit 2017/18. London, Royal College of Physicians, 2019.

40 O'Driscoll BR, Howard LS, Davison AG. BTS guideline for emergency oxygen use in adult patients. Thorax 2008; 63: Suppl. 6, vil-v68.

41 Yang IA, Brown JL, George J, et al. The COPD-X Plan: Australian and New Zealand Guidelines for the management of Chronic Obstructive Pulmonary Disease 2019. Brisbane, Lung Foundation Australia, 2019.

42 Puhan MA, Gimeno-Santos E, Cates CJ, et al. Pulmonary rehabilitation following exacerbations of chronic obstructive pulmonary disease. Cochrane Database Syst Rev 2016; 12: CD005305.

43 Alison JA, McKeough ZJ, Johnston K, et al. Australian and New Zealand pulmonary rehabilitation guidelines. Respirology 2017; 22: 800-819.

44 Ospina MB, Mrklas K, Deuchar L, et al. A systematic review of the effectiveness of discharge care bundles for patients with COPD. Thorax 2017; 72: 31-39. 